\title{
DiOTTOS
}

\section{Diez años de redes sociales $y$ periodismo: riesgos $y$ retos en el ecosistema informativo del s. XXI ${ }^{1}$ \\ Ten years of social networks and journalism: risks and challenges in the information ecosystem of the 21st century}

Recibido: 2018-18-14

Aceptado: 2019-04-25

\author{
Salvador Enguix \\ salvador.enguix@uv.es \\ Universitat de València
}

\section{Resumen}

En este trabajo presentamos, a modo de panorámica crítica, un análisis sobre lo que ha supuesto la irrupción y asentamiento de las redes sociales en el ámbito periodístico en los últimos 10 años. Analizamos, en primer lugar, cómo el propio periodismo se hace eco de este fenómeno, convirtiéndolo en objeto noticiable; esta cobertura se realiza en los primeros años con cierto optimismo ingenuo, que progresivamente se va tornando en discurso de crítica, especialmente desde el escándalo de Facebook y Cambridge Analytica en 2018. En segundo lugar, consideramos los dos aspectos positivos que, todavía, puede suponer el uso de las redes sociales para el periodismo: el incremento indudable de la difusión de los contenidos periodísticos y la ayuda en la creación de la marca personal del periodista. Por último, revisamos los factores que sí pueden tener consecuencias negativas, no solo para los medios, sino para su función natural en las sociedades democráticas: las redes sociales no crean

\begin{abstract}
In this work, a critical overview about the irruption and consolidation of social networks in the journalistic field in the last 10 years is presented. We analyze, in the first place, how journalism itself echoes this phenomenon, turning it into a newsworthy object. In the initial years this coverage is carried out with a certain naive optimism, which progressively becomes a critical discourse, especially since the scandal of Facebook and Cambridge Analytica in 2018. Second, we consider the two positive aspects that can still be assumed in the use of social media journalism: the undoubted increase in the dissemination of journalistic content and the help in the creation of the journalist's personal brand. Finally, we review the factors that can have negative consequences, not only for the media, but also for their natural function in democratic societies: social networks do not create content, but use content generated (financed) by the media; its dynamics of work interfere in the professional routines
\end{abstract}

\footnotetext{
${ }^{1}$ Proyecto de investigación PRODIS-NET: Procesos discursivos en internet: confluencia de partidos, medios y ciudadanos, Ref. FFI2015-67668-R, del Ministerio de Economía y Competitividad y con financiación FEDER.
} 
Enguix, S. (2019).

Diez años de redes sociales y periodismo: riesgos y retos en el ecosistema informativo del s. XXI pp. 164/183

contenidos, sino que utilizan contenidos generados (financiados) por los medios; sus dinámicas de funcionamiento interfieren en las rutinas profesionales del periodismo, especialmente en la producción de contenidos; desafían el modelo de negocio tradicional porque acaparan la inversión publicitaria; y por último, intervienen y condicionan el desarrollo de la agenda informativa.

\section{Palabras clave:}

Redes sociales. Periodismo. Rutinas profesionales. Facebook. Twitter. of journalism; they challenge the traditional business model because they monopolize the advertising investment; finally, they intervene and condition the informative agenda-setting

\section{Introducción: las redes sociales relevantes para el periodismo}

Aunque existen muchas redes sociales (RRSS), es evidente que las más relevantes para el ecosistema informativo y, por extensión, el periodismo político, son, fundamentalmente, Twitter (TW) y Facebook (FB), por su capacidad para generar tráfico en las ediciones digitales, y en menor medida también Instagram. Se trata de las redes de mayor difusión y alcance, y por ello son las que tienen un impacto más constante y decisivo en la configuración del espacio público. Desde su aparición (Facebook en 2004, Twitter en 2006, Instagram en 2010), estas redes han ido adquiriendo una presencia cada vez mayor en el espacio comunicativo general, sin que el ámbito periodístico y la difusión de noticias sea una excepción. Pero, tal y como hemos señalado en otro lugar (Autor 2017), las plataformas de redes sociales, y por tanto las empresas propietarias, admiten una doble consideración, en la medida en que son herramientas de comunicación horizontal entre iguales pero también actúan como fuentes de difusión vertical de contenidos (ajenos o creados por los usuarios). A partir de estas dos funciones básicas es decir, como social networks y como social media - es posible identificar para estas empresas hasta cinco grandes funciones:

- Creación de comunidad, que en una dimensión política/informativa puede presentarse como refuerzo de la democracia (experiencias emblemáticas como la Primavera árabe, o el 15M).

- Difusión de contenidos sin necesidad de intermediación, lo que en términos políticos se alinea con la libertad de expresión, dando voz a colectivos y personas que no obtienen atención de los medios. 
Enguix, S. (2019).

Diez años de redes sociales y periodismo: riesgos y retos en el ecosistema informativo del s. XXI pp. 164/183

- Filtro de contenidos y censura, pues las redes silencian y borran la aportación de múltiples usuarios, y modifican parámetros de difusión con sus algoritmos.

- Jerarquización de contenidos, pues los algoritmos de ordenación de las publicaciones intervienen el establecimiento de la agenda.

- Intervención directa en la financiación de partidos políticos en países que lo permiten, mediante sus Comités de Acción Política (PAC).

El análisis de estos cinco ámbitos de actuación permite entender las empresas de RRSS como verdaderos actores políticos en el sentido clásico que propusiera Héctor Borrat para los periódicos (Borrat 1989). Así, aunque sus directivos gustan de señalar que se trata de herramientas de comunicación cuya finalidad básica es “crear comunidad” y desarrollar tecnologías complejas e innovadoras - y esto es especialmente relevante en el caso de Facebook, por el protagonismo tanto mediático como empresarial de Zuckerberg (Salmon 2016)_, ya nadie niega el papel que estas corporaciones han adquirido en el plano informativo y político de nuestras sociedades. Esta capacidad de intervención las acerca al papel tradicional de los medios de comunicación, pero, tal y como señalan las críticas crecientes —a veces desde los mismos medios (Applebaum 2015; Bell 2014, 2016; Greenslade 2016; Greensland 2016)_-, sin asumir la responsabilidad de rendición de cuentas que sí tienen las empresas informativas (Gallardo y Enguix 2016: 161).

Los escándalos protagonizados por Facebook han sido especialmente difundidos entre 2016 y 2018, pero es necesario señalar que Twitter tampoco escapa a este tipo de polémicas (Theocharis et al. 2016). Situaciones como la venta de datos privados de los usuarios, el engaño a adolescentes para comprar aplicaciones que espiaban sus móviles, o la participación acrítica en la difusión y viralización de noticias falsas y discursos antidemocráticos, son ejemplos de los diversos fenómenos que han puesto de manifiesto (Isaak y Hanna 2018) la incapacidad de gobiernos e instituciones para anticipar el impacto de estas tecnologías comunicativas. Se suma además la importancia que ha adquirido la red social GAB en la difusión de los mensajes racistas, xenófobos y supremacistas blancos de la alt-right estadounidense (Reguera 2017; Daniels 2018; Liddle 2016). Las redes han asumido funciones que van mucho más allá de la conexión interpersonal y la expresión individual, y varias de esas funciones entran en colisión directa con el papel tradicional que nuestras sociedades concedían a la prensa y al periodismo político desde el siglo XIX.

En este trabajo ofrecemos una revisión sobre la relación entre estas plataformas de comunicación digital y los medios de comunicación, especialmente la prensa escrita y en su versión digital. Creemos que los años transcurridos desde la irrupción de las redes sociales permiten ya esbozar una mirada panorámica global, y revisar cuál ha sido su incidencia en el periodismo, señalando luces y sombras que, en gran medida, siguen manteniendo una tensión complicada. Para estructurar este repaso bibliográfico atenderemos a cuatro aspectos: 
Enguix, S. (2019).

Diez años de redes sociales y periodismo: riesgos y retos en el ecosistema informativo del s. XXI pp. 164/183

- Twitter y Facebook como fenómenos noticiables

- Las redes sociales como oportunidad para los medios de comunicación

- Las redes sociales como desafío para el periodismo político

- Nuevos actores: las aplicaciones de mensajería rápida.

Nuestra premisa fundamental es que los medios de comunicación reaccionaron en los primeros años con un entusiasmo acrítico que no supo prever hasta qué punto estas empresas podían convertirse en auténticas competidoras. La evolución de las conductas comunicativas desempeñadas en dicho entorno, tanto por los medios de comunicación como por los falsos medios y los ciudadanos individuales, se ha convertido en un verdadero desafío para el periodismo y, en buena lógica, para su función en las sociedades democráticas. Clavero (2018: 178) señala en este sentido que "el periodismo parece no encontrar su lugar de relevancia en el diálogo permanente que los ciudadanos tienen consigo mismos y con las instituciones”.

\section{Facebook y Twitter como noticia}

Una de las primeras señales de la importancia concedida por los medios de comunicación a las redes sociales ha sido su conversión en enganche informativo: los medios se hacen eco de lo que ocurre en estos ámbitos con una atención que no conceden a otro tipo de contextos comunicativos. Así, en una encuesta realizada a 50 periodistas políticos españoles en 2017 (Autor 2019), a la que respondieron 34 de ellos, un 29\% señalaba que sus medios tenían una sección específica para hacerse eco de los temas tratados en redes sociales. La atención de los periódicos se refleja en titulares como los siguientes, pertenecientes a los primeros años:

- Facebook: De fenómeno social a tesis académica (El País, 14/01/2008).

- La campaña en la red. Facebook opina sobre los políticos (El País, 28/02/2008).

- Twitter es la herramienta de la inmediatez y la cercanía (La Vanguardia, 08/03/2008).

- Israel pide a EE.UU. que ataque con Twitter y Facebook (H. Cymerman, La Vanguardia, 08/12/2009)

- Un pasajero retransmite desde su Twitter el accidente del avión en el que viajaba (ABC, 22/12/2008)

- El PP afina el perfil de Rajoy en Facebook (El País, 22/01/2009)

- “Tuenti” y “Facebook” registran miles de adhesiones (ABC, 27/01/2009)

- Redes sociales, un saco sin fondo de intimidades (El Mundo, 23/02/2009)

- Redes sociales. Facebook cede parte del control a los usuarios (El País, 28/02/2009)

Además, Facebook y Twitter se utilizan también como fuente para citar las declaraciones de los representantes políticos u otros protagonistas de la actualidad informativa, con lo que se produce la paradoja de que los medios están sirviendo de plataforma de difusión para lo que dicen los usuarios de las redes sociales. 


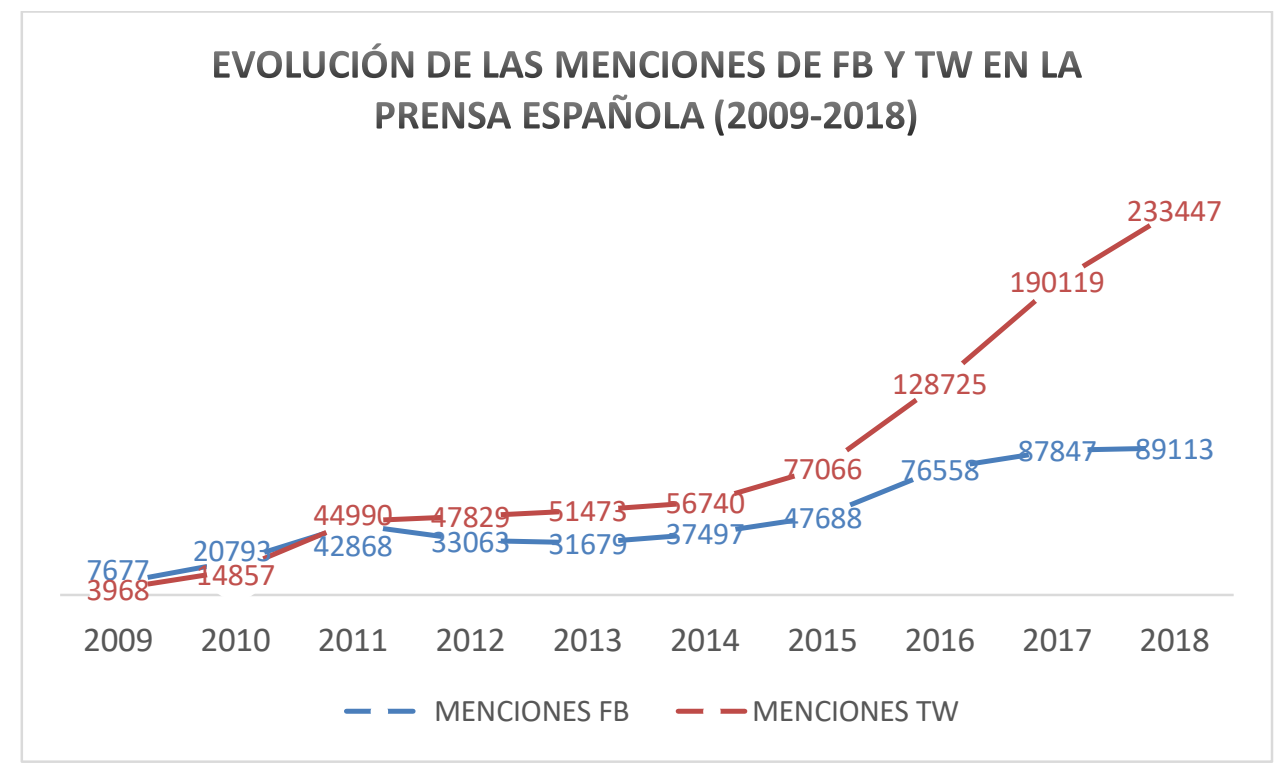

Gráfico 1. La presencia de Facebook y Twitter en los medios españoles entre 2009 y 2018. Fuente: FACTIVA, elaboración propia.

En un segundo momento, la búsqueda se redujo generando dos tipos de información relativos a:

- Fuentes: para cada anualidad, se separaron los datos concretos referidos a los diarios generalistas El País, El Mundo, ABC y La Vanguardia.

- Ámbito temático: para cada año se obtuvo también el análisis temático en el que la plataforma tiene almacenado cada documento.

Tal y como señala el gráfico 1, el análisis global de la presencia de FB y TW en los medios impresos españoles (incluyendo tanto agencias como diarios) ofrece un claro predominio de Twitter, contrariamente a lo que observaremos más tarde respecto a cómo actúan las dos redes en la difusión de noticias digitales. Esta situación general se refleja también en los cuatro periódicos impresos que el EGM suele recoger como los de mayor tirada.

\subsection{La progresiva visibilidad de las redes en la prensa escrita}

El gráfico 2 muestra un subcorpus referido a cómo los periódicos generalistas de mayor tirada (El País, El Mundo, ABC y La Vanguardia) se han hecho eco del fenómeno y han contribuido a darle valor noticiable; el incremento es incesante en todos los medios. El número total de piezas periodísticas que mencionan una de las dos redes sociales en los cuatro rotativos durante los 10 años suma 129.019 textos. 
Enguix, S. (2019).

Diez años de redes sociales y periodismo: riesgos y retos en el ecosistema informativo del s. XXI pp. $164 / 183$

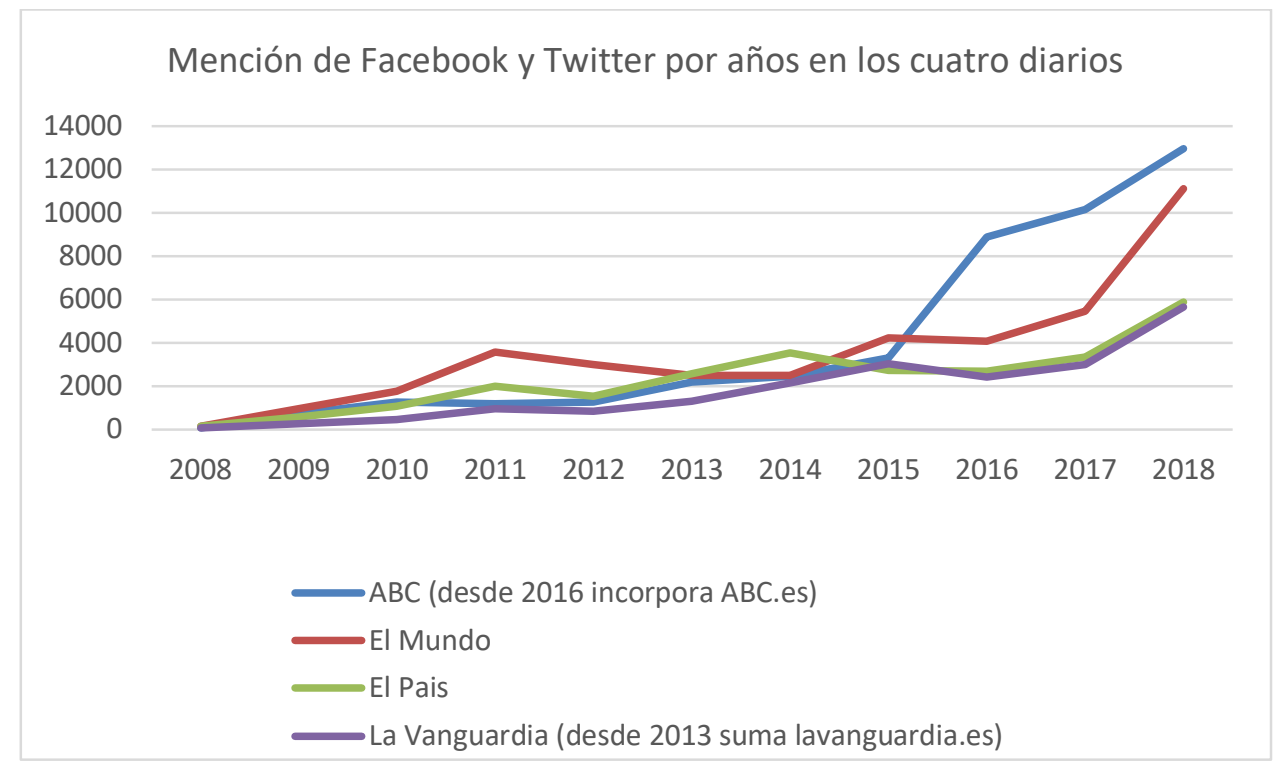

Gráfico 2. Evolución de las menciones de redes sociales en los distintos medios impresos durante los últimos 10 años. Fuente: FACTIVA, elaboración propia.

Los medios, pues, han contribuido a incrementar la importancia que las redes sociales iban ganando en nuestras sociedades, legitimándolas como focos relevantes de opinión pública.

\subsection{Twitter y Facebook en noticias de naturaleza política}

Además de servir como plataformas ecoicas para la importancia creciente de las redes sociales, la prensa ha contribuido también a reforzar su incidencia específica en el ámbito del periodismo político y la política.

La bibliografía sobre el uso de Facebook y Twitter por parte de representantes e instituciones políticas insiste en el fenómeno de la desintermediación, es decir, la posibilidad de acceder al flujo del discurso público sin necesitar la implicación de los medios de comunicación; estos cambios producirían alteraciones en las relaciones de poder de la comunicación política (Broersma y Graham 2016; Bobba 2018). Pero esta afirmación puede matizarse, ya que la desintermediación no viene dada por las redes sociales digitales, sino por la tecnología de Internet, de manera que todos los partidos políticos y los líderes pueden disponer de acceso directo (no mediatizado) al discurso público mediante páginas web y blogs, sin recurrir a las plataformas de redes. Lo que estas aportan, además, es una interfaz muy concreta que crea una falsa sensación de diálogo, una utópica versión digital de ágora política que se asumió sin cuestionamiento, confundiendo el encadenamiento sucesivo de mensajes con la interacción deliberativa; no en vano Bell (2016) propone hablar de reintermediación, en lugar de desintermediación. 
Enguix, S. (2019).

Diez años de redes sociales y periodismo: riesgos y retos en el ecosistema informativo del s. XXI pp. 164/183

En efecto, y tal y como ha destacado Gallardo (2018: 65-66), las redes sociales emergieron como una herramienta de aparente democratización discursiva que culminaba la presunta horizontalidad de la red, es decir, el hecho de que todos los usuarios se encuentran en igualdad de condiciones para expresarse. Pero los estudios específicos sobre la existencia de interacción real entre los perfiles de las redes sociales, pese a las predicciones optimistas (Tumasjan et al. 2010), muestran que esta es mínima, casi testimonial; los escasos ejemplos de políticos que responden a la interpelación de los usuarios (Broesma y Graham 2016; Gallardo y Enguix 2016) se dan en perfiles de redes gestionados por los equipos de comunicación del político o, incluso, mediante el recurso a bots de inteligencia artificial (Sanchez Gonzales y Sánchez González 2017).

Aunque la cantidad de piezas periodísticas que aluden a las RRSS en los diez años no es manejable en un análisis detallado, FACTIVA nos ofrece información sobre el ámbito periodístico general al que se adscribe cada texto. Por tanto, descargamos en cada anualidad los metadatos referidos al tema global en el que se enmarcan las menciones de la búsqueda léxica, a fin de averiguar cuándo la mención de Twitter y Facebook tenía relación con las noticias de naturaleza política.

FACTIVA propone un listado de cien ámbitos informativos para etiquetar los resultados de cada búsqueda. De ellos, consideramos que eran de relevancia política los siguientes: Ministerio de Defensa, Política nacional, Noticias económicas, Ministerio de Educación, Elecciones, Unión Europea/Parlamento Europeo, Poder ejecutivo, Poder legislativo, Poder judicial, Presupuesto del gobierno, Impuestos, Ministerio del interior, Relaciones/política internacional, Política, Medidas gubernamentales, Política autonómica, Congreso/Senado; no hemos tenido en cuenta la inclusión de otras esferas informativas periféricas a la política de la última década, como por ejemplo aquellos casos en que las menciones de Facebook o Twitter tenían lugar en textos relacionados con las Fuerzas Armadas, los movimientos civiles y los derechos humanos, la corrupción o las noticias relacionadas con terrorismo. 
Enguix, S. (2019).

Diez años de redes sociales y periodismo: riesgos y retos en el ecosistema informativo del s. XXI pp. 164/183

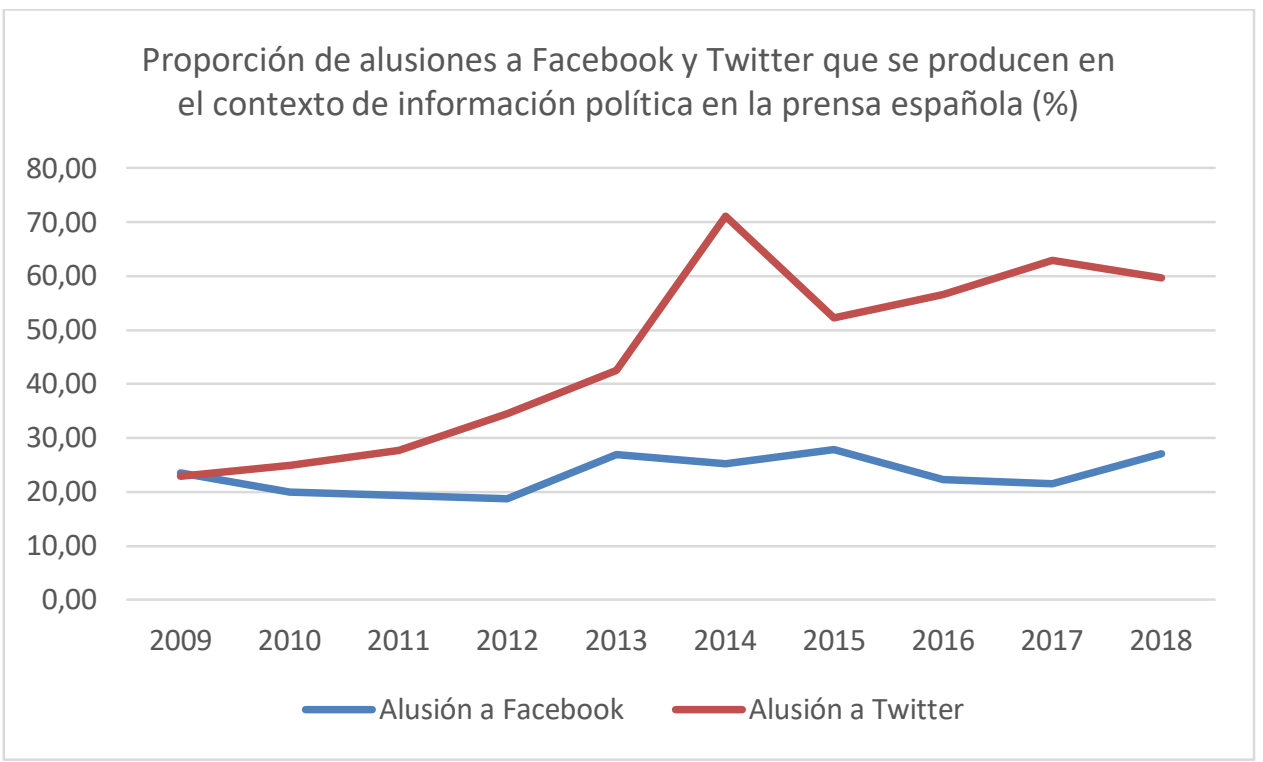

Gráfico 3. Menciones de Facebook y Twitter de la prensa española que se producen en noticias de ámbito político. Fuente: FACTIVA, elaboración propia.

El gráfico 3 muestra cómo la prensa ha otorgado también en la esfera política más relevancia a Twitter que a Facebook, con una diferencia que se mantienen bastante estable a lo largo de los años; desde los años iniciales, las menciones de las redes sociales se producen en noticias políticas, dándose en 2014 la máxima relación, año en que los datos indican que un $71 \%$ de las alusiones a Twitter se producían en noticias políticas o con protagonistas del mundo de la política:

- Cameron critica una página en 'Facebook' de apoyo al fugitivo que se suicidó (El Mundo, 14/07/2010).

- Los internautas denuncian el "\#Prostituit" del PP (El País, 06/10/2011).

- Elena Valenciano deja Twitter por la "persecución” que sufren sus hijos (El País, 01/03/2013).

- Lo mejor de la reacción de Twitter a la abdicación del Rey (El País, 02/06/2014).

- \#NoPodéis o \#PodemosConEllos: las redes sociales, contra el partido de Pablo Iglesias (ABC, 02/02/2015).

- Twitter expulsa definitivamente al ultra Alex Jones (El País, 07/09/2018).

- La semana de DiosTuitero: un paseo por el mundo de la política y la Familia Real (El Mundo, 28/01/2018).

- Bruselas "pierde la paciencia" con Facebook: "Es un flujo de basura y odio" (El Mundo, 20/09/2018).

- Europa quiere morder más fuerte: pide una auditoría a Facebook para evaluar su seguridad (ABC, 29/10/2018).

Los periodistas introducen estas alusiones en sus textos para hacerse eco del impacto que obtienen en la red ciertos hechos o, sobre todo, ciertos mensajes de los actores políticos. 
Enguix, S. (2019).

Diez años de redes sociales y periodismo: riesgos y retos en el ecosistema informativo del s. XXI pp. 164/183

Esta actitud de la prensa ha contribuido sin duda a asentar la falacia de que las redes sociales son un reflejo directo de nuestras sociedades, aunque los análisis detallados (Gallardo 2017) muestren que no hay coincidencia entre los discursos de los usuarios y los resultados electorales.

Una vez producida la integración de las redes sociales en las dinámicas comunicativas públicas y privadas de nuestras sociedades, nos planteamos en los apartados siguientes una panorámica global sobre su impacto en el desempeño del periodismo político.

\section{Las redes como oportunidad para el periodismo}

Como hemos adelantado, la aparición de las redes sociales fue recibida por los medios con un notable entusiasmo; los primeros estudios (Ahmad 2010; Kwak et al. 2010; Hermida 2010) destacaban la confianza acrítica del periodismo en las nuevas tecnologías. Ahmad (2010: 148), por ejemplo, hablaba de una confianza "evangélica” de los periodistas en las redes sociales.

Desde el punto de vista teórico, esta visión nos lleva desde la posición integrada de los planteamientos clásicos de Eco (1968) hasta la moderna teoría de la normalización (Lasorsa, Lewis, Seth y Holton 2012). Para Umberto Eco, las visiones apocalípticas surgían en los textos sobre la comunicación de masas (especialmente los basados en la tradición de Adorno y la Escuela de Franckfurt), mientras que las posiciones integradas eran visibles sobre todo en los textos surgidos de la comunicación de masas (Eco 1968: 12). Paralelamente, la teoría de la normalización intenta dar cuenta de cómo los periodistas van adaptando de una manera bastante fluida las posibilidades tecnológicas que ofrece la digitalización a los parámetros y valores procedentes del mundo predigital (objetividad, imparcialidad). Es decir, cuando los profesionales se enfrentan a nuevas herramientas como las redes sociales, van adaptándolas a sus necesidades, y naturalizan progresivamente su uso (Parmelee 2014; Revers 2014; Hedman 2015). No es extraño que, en la encuesta antes referida (Autor 2019), la totalidad de los participantes declare tener perfil activo en Twitter y un 93\% de ellos en Facebook, y que el mantenimiento de tales perfiles se haya convertido en una rutina profesional.

Como huella de esta normalización, existen al menos dos aspectos en los que podemos considerar que las redes sociales suponen (o al menos, han supuesto) una oportunidad para el periodismo:

1. En primer lugar, desde el punto de vista de los medios, las redes sociales se convierten en una oportunidad de difusión de las versiones digitales de sus contenidos.

2. En segundo lugar, las redes son importantes en la medida en que ayudan al periodista a desarrollar su marca personal. 
Enguix, S. (2019).

Diez años de redes sociales y periodismo: riesgos y retos en el ecosistema informativo del s. XXI pp. $164 / 183$

\subsection{Las redes amplían la difusión de los contenidos periodísticos}

Para el año 2015, un estudio de Kantar (Nafría 2015) señalaba que el 53,8\% de los españoles buscaba la información política básicamente en la televisión frente a un 21,9\% que elige la red como medio primario; de este grupo, un 46,3\% recurría preferentemente a los perfiles seguidos en Facebook y Twitter. Más recientemente, los datos de España del informe Reuters (Digital News Report 2018, Newman et al. 2018: 10), señalan que un $48 \%$ de los encuestados declara utilizar Facebook como fuente de noticias, seguido por WhatsApp (un 36\%), Youtube (un 26\%) y, en cuarta posición, Twitter (un 22\%); respecto a las cifras de 2017, estos datos suponen un aumento del 1\% en el caso de Facebook, y del 4\% tanto para YouTube como para Twitter.

Estas cifras indican que los medios reciben gran parte del tráfico que llega a sus sitios web a partir de enlaces en las redes sociales; teniendo en cuenta que la financiación por publicidad depende del número de visitas que tenga cada página web, es fácil entender que los periódicos estén interesados en mantener y aumentar este flujo de comunicación.

\subsection{Las redes facilitan la construcción de la marca personal del periodista}

En otro orden de cosas, las redes han contribuido notablemente a que los periodistas (especialmente en el ámbito del periodismo político y el periodismo deportivo) desarrollen su marca personal, en un sentido que no coincide exactamente con el antiguo concepto de "periodista estrella” (Carrera et al. 2012), sino que busca individualizarse como voz específica en el ecosistema informativo. Las redes, efectivamente, distribuyen la visibilidad de sus usuarios y se han convertido en fuente de noticiabilidad, hasta el punto de que se crea una especie de paradoja circular en la que no es que las redes se hagan eco de la actualidad, sino que alguien/algo adquiere esa actualidad precisamente por estar en redes:

Resulta especialmente interesante, de hecho, observar cómo algunos de los colaboradores periodísticos más recientemente incorporados, dirigentes políticos de nuevo cuño o líderes de opinión en cualquier sector (económico, artístico, religioso, etcétera), no solo tienen muy presente su actividad en las redes sociales como parte de su estrategia de comunicación y de intervención en el espacio público, sino que, a menudo, es justamente su notoriedad y su presencia en las redes sociales la que está detrás de buena parte de su éxito. Las redes sociales, en este sentido, no solo reflejan las tendencias de fondo, los liderazgos y los debates que se producen en la sociedad, sino que también contribuyen a crearlos. (López García 2017b: 233).

Este rasgo se alinea, sin duda, con el personalismo que caracteriza a su vez el discurso de la política (Gallardo y Enguix 2016), en el que los hiperliderazgos han servido para alentar las retóricas populistas de los últimos años, y las redes han servido de plataforma para el exhibicionismo ideológico (López García 2017a: 577).

Hedman y Djerf-Pierre (2013: 371) señalan que los perfiles de los periodistas en las redes sociales contribuyen, por un lado, a la creación de marca corporativa, pero por otro, 
Enguix, S. (2019).

Diez años de redes sociales y periodismo: riesgos y retos en el ecosistema informativo del s. XXI pp. $164 / 183$

permiten también el desarrollo de la marca persona; los usuarios de redes sociales declaran preferir a aquellos periodistas que transgreden las exigencias de neutralidad profesional y utilizan Twitter para manifestar sus opiniones políticas personales respecto a las noticias que publican en sus medios. Estas autoras identifican tres perfiles básicos de periodista en las redes sociales:

1. Los ermitaños escépticos, periodistas que evitan tener nada que ver con las redes.

2. Los conformistas pragmáticos, periodistas que usan regularmente las RRSS, básicamente para recopilar información y pulsar la actualidad; no les preocupa la marca personal digital y no aceptan el borrado de límites entre vida personal y vida profesional.

3. Los activistas entusiastas, son periodistas que están conectados continuamente a internet a y las redes sociales, que utilizan más allá de la información y las funciones de escaneo ambiental, y con frecuencia utilizan las redes sociales para la creación de redes, la marca personal y la colaboración.

\section{Las redes como reto para el periodismo}

\subsection{Las redes vampirizan los contenidos periodísticos}

En un artículo sobre la utilidad de Twitter para el periodismo, ya en el año 2010, Ahmad se planteaba qué tipo de capital, social y económico, aportaba Twitter a los periodistas, y concluía que en realidad son los medios los que están al servicio de las redes, pues son las grandes empresas tecnológicas las que controlan los beneficios generados en la red.

Ahmad hablaba de la forma "desproblematizada y festiva" en que muchos periodistas concebían las RRSS, como "nuevos dioses" de un nuevo ecosistema informativo (2010: 48), y la rapidez con la que los profesionales (directores, redactores, editores) se abrieron un perfil en las plataformas (Fernández y Vázquez 2016). Este optimismo, al que ya nos hemos referido, facilitó en los años iniciales la consolidación de las plataformas de redes sociales, con la ayuda de diversas apps (Bell 2016) que estas empresas ponían a disposición de periodistas y editores, como beneficiar a periodistas y editores, con apps como Discover (Snapchat), Instant Articles (Facebook), Apple News y Accelerated Mobile Pages (Apple), Moments (Twitter).

\subsection{Las redes interfieren en las rutinas profesionales y en los valores del periodismo}

Las redes han afectado poderosamente el ritmo de trabajo de los periodistas y de construcción de las noticias, básicamente debido a factores como la velocidad y la constancia, es decir, el hecho de que los ciclos de noticias se han convertido en ciclos de 24 horas, mucho más allá de lo que supuso la aparición de los canales de televisión por cable.

Muchas de las características que han ido definiendo las redes sociales y su dinámica de funcionamiento encierran alguna contradicción o cuestionamiento de los estándares y 
Enguix, S. (2019).

Diez años de redes sociales y periodismo: riesgos y retos en el ecosistema informativo del s. XXI pp. 164/183

valores normalmente asociados al periodismo. Algunas de estas contradicciones emergen en una encuesta a periodistas políticos españoles que hemos mencionado ya (Autor, 2019); por ejemplo, un 38\% de los periodistas admitía publicar opinión propia sobre los temas de actualidad, no solo información o enlace a los textos de su medio. En lo referente a la credibilidad de las publicaciones, mientras un 65\% decía que no se fiaba de las redes sociales como medio de verificación y un 73\% declaraba explícitamente que no concedía credibilidad sistemática a los mensajes, un 21\% admitía haber difundido alguna vez alguna noticia falsa y darse cuenta más tarde. Estas contradicciones parecen apuntar a la idea de que los periodistas mantienen un concepto clásico de cuál es su función social, política e informativa, es decir, su profesión, pero las redes sociales desafían este concepto en la praxis. La relación entre redes sociales y credibilidad de la prensa es importante por la decisiva contribución de estas plataformas en la difusión de las noticias falsas; la paradoja ya señalada por Bennett e Iyengar (2008: 714), según la cual los ciudadanos asumen cada vez más la relevancia de los medios de comunicación en la esfera política, pero les conceden cada vez menos credibilidad.

\subsection{Las redes desafían el modelo de negocio tradicional de los medios impresos}

Para el año 2017 se calcula que Facebook ganó, como promedio, 20,21 dólares por cada usuario, y Twitter 7,44 dólares por cada usuario (Pérez 2018). La mayoría de estos ingresos ARPU (Average Revenue Per User) procede de la publicidad, una publicidad que en el mundo analógico se concentraba en los medios de comunicación y facilitaba su financiación.

Efectivamente, las redes sociales han contribuido al final del modelo de negocio de los medios de comunicación que se inauguró cuando, en 1883, el director del New York Sun, Benjamin Day, decidió abaratar los costes del periódico introduciendo publicidad en sus páginas (Del Fresno, 2015: 107); desde entonces, los medios impresos han tenido dos vías fundamentales de financiación: la venta del propio diario y los ingresos de publicidad.

Desde la economía política de la comunicación son muchos los autores que, como CaseroRipollés (2010, 2012), han señalado la necesidad de repensar el modelo de negocio de la prensa por la competencia que suponía Internet, incluso en tiempos previos a la consolidación de las redes sociales:

La publicidad genera unos ingresos insuficientes para asegurar la viabilidad de la prensa en internet. Este hecho está orientando las estrategias de las empresas periodísticas hacia la implantación de sistemas de pago por el acceso a los contenidos informativos. En la actual coyuntura, donde predomina el ensayo-error, parece que las fórmulas mixtas, como el freemium, que combinan cobro y gratuidad, se alzan como la mejor solución. Mientras asistimos a su afianzamiento o a su fracaso, la ausencia de nuevos modelos de negocio solventes no sólo pone en aprietos el futuro de la prensa en internet sino que complica la consolidación de nuevos proyectos periodísticos en la Red. (Casero-Ripollés 2010: 600$601)$. 
Enguix, S. (2019).

Diez años de redes sociales y periodismo: riesgos y retos en el ecosistema informativo del s. XXI pp. 164/183

Han pasado casi diez años desde estas reflexiones, durante los que hemos asistido a la irrupción de las redes sociales, pero ese nuevo modelo de negocio se resiste a cuajar. No obstante, existen fórmulas de pago que parecen respaldar cierto optimismo de futuro. En un trabajo más reciente, Palacio (2018) ha descrito la aparición de medios como El Confidencial (2001), Valencia Plaza (2010), eldiario.es (2012), o Infolibre (2013), que normalmente combinan el formato digital con apoyo de algún medio impreso (Revista Plaza, Tinta Libre...):

En los medios que se dirigen a mercados locales, la situación es ligeramente diferente, en el sentido de que los recursos están más limitados; lo cual, en ocasiones, obliga a buscar un mayor número de fuentes de ingresos. Es el caso de Valencia Plaza, un medio especializado en la economía valenciana que en sus orígenes trató de financiarse con recursos publicitarios. Según su director, Javier Alfonso, poco después del lanzamiento quedó claro que los precios de la publicidad digital hacían inviable el proyecto; y, cuatro años después, en 2014, se lanzó la revista cultural impresa, Plaza, que comenzó a aportar recursos que ayudaron a ir estabilizando las finanzas de la compañía, que hasta ese momento se había mantenido gracias a las aportaciones de los accionistas. Con posterioridad se lanzaron otras publicaciones, como la Guía Hedonista, dirigida al mercado de la restauración y los productos gourmet. "Pero, sobre todo -explica Alfonso-, se inició la organización de eventos patrocinados, de los que ya se celebran unos ocho cada mes". Todas esas actividades llevaron los ingresos del grupo Ediciones Plaza hasta 1,8 millones en 2017, y la compañía ha iniciado la expansión a las provincias limítrofes (Palacio 2018: 21).

Las suscripciones son la otra opción destacable para la financiación de los medios. Aunque la situación no es extrapolable a todos los diarios y países (la acritud del presidente Donald Trump hacia la prensa y, en concreto, hacia el NYT se suele mencionar como un factor que ha provocado el incremento de suscriptores de este periódico durante su mandato), Peiser (2019) refiere que The New York Times consiguió en 2018 unas ganancias de 709 millones de dólares por ingresos de la publicidad digital, gracias al modelo de suscripción que ha desarrollado, y según el cual prevé una cifra de 10 millones de suscriptores para 2025:

More than 3.3 million people pay for the company's digital products, including its news, crossword and food apps, a 27 percent jump from 2017. The total number of paid subscriptions for digital and print reached 4.3 million. Online subscription revenue gained nearly 18 percent to reach $\$ 400$ million in 2018, while digital advertising rose 8.6 percent, to $\$ 259$ million. In the last three months of the year, digital subscription sales grew at a slower pace, about 9 percent, to $\$ 105$ million. That slowdown came partly as a result of marketing efforts to draw more paying readers. The Times has offered introductory discounts for online access, which attracts new readers who bring in less revenue. Over time, the company expects many of them to become full subscribers. (Peiser 2019).

\subsection{Las redes intervienen en la agenda y contribuyen a la difusión viral de noticias falsas}

Junto al impacto estricto de las RRSS en el ámbito profesional del periodismo, cabe plantearse también cómo ese impacto puede tener un efecto rebote que boicotea el papel del periodismo en las sociedades democráticas. Cabe pensar que cuando estas empresas, 
gracias a sus posibilidades tecnológicas (Maréchal 2016), parecen desempeñar funciones propias de la prensa, interfieren con el papel que las sociedades democráticas conceden a este actor social. Este es sin duda el factor más frecuente en las críticas destinadas a Facebook y Twitter.

$\mathrm{Al}$ asumir funciones propias de los medios de comunicación, pero sin respetar garantías como la verificación de fuentes o la comprobación de datos, las redes sociales contribuyen a la polarización política e intervienen en la agenda informativa — como ya hemos señalado, muchas veces con la complicidad de los medios de comunicación. De este modo, desafían el papel tradicional de los medios como guardabarreras de la información (Broesma y Graham 2016: 90). Los estudios sobre la incidencia de Facebook y Twitter en la difusión de falsas noticias son cada vez más. Por ejemplo, Clavero (2018) subraya cómo los algoritmos favorecen la opacidad y la difusión de noticias falsas; refiere el análisis de Guess et al. (2018) sobre su importancia en las elecciones que dieron la victoria a Trump:

Durante las elecciones presidenciales en Estados Unidos, el 27,4\% de los americanos de 18 años o más visitó algún sitio pro-Clinton o pro-Trump de fake news. Pero estos datos se tornan más significativos cuando, de las personas encuestadas que se muestran propensas a visitar sitios web de fake news a favor de su candidato preferido, solo el 63\% afirma tener una visión "muy" o "algo favorable" de sitios o páginas que se dedican a la verificación de los hechos (pp. 5-10), lo que expresa un relativo interés por la veracidad de la información. (Clavero, 2018: 171).

Es decir: todas estas actuaciones han alterado decisivamente la percepción general sobre las redes sociales, y el entusiasmo de los primeros años se ha ido trastocando en una suerte de pesimismo resignado, que se plasma en artículos de opinión donde algunos periodistas recomiendan abandonar la red social o limitar su uso a una presencia de observación atenta que podríamos denominar “de escaparate”. Por ejemplo, en enero de 2019 Farhad Majoo publicaba una columna en The New York Times titulada con la tajante sentencia "Periodista, es el momento de dejar Twitter":

A diario, la red social favorita de los medios sumerge cada vez más a los periodistas en el melodrama tribal, lo cual hace cortocircuito con nuestros mejores instintos y favorece el pensamiento de grupo basado en los bots y las masas. Durante este proceso, se ayuda a aumentar los estereotipos más dañinos de nuestra profesión. En vez de volverlos cronistas curiosos e intelectualmente honestos de los asuntos de la humanidad, Twitter constantemente convierte a muchas personas de los medios - me incluyo - en bots irracionales de la indignación que reaccionan por instinto después de adoptar tal o cual causa llena de etiquetas, misivas presidenciales mal escritas o campañas de información dirigida. Sin embargo, Twitter no solo arruina la imagen de los medios. También sesga nuestro periodismo. Todos los elementos de la interfaz de Twitter fomentan una mentalidad que se opone a la indagación periodística: favorece la forma por encima del contenido y los argumentos baratos por encima del debate razonado mientras reduce el alcance temporal de la prensa. (Majoo, 2019). 
Enguix, S. (2019).

Diez años de redes sociales y periodismo: riesgos y retos en el ecosistema informativo del s. XXI pp. 164/183

La alusión a la interfaz del último párrafo es importante; efectivamente, la disposición gráfica del encadenamiento de mensajes, la limitación del espacio de la pantalla, la dinámica de deslizamiento rápido de la pantalla... son condicionantes tecnológicos que influyen en la comunicación y los procesos cognitivos asociados (Gallardo 2018), e imponen una velocidad de consumo de noticias y una inmediatez que contradicen algunos de los valores periodísticos fundamentales. Sin embargo, resulta igualmente difícil eludir la realidad que suponen las redes sociales y sus aportaciones positivas, a las que nos hemos referido en el apartado previo. El día siguiente a la columna de Majoo, era Jeff Jarvis, bien conocido por su defensa de la información en Internet, quien salía en defensa de las redes sociales como conversación activa de los periodistas con la ciudadanía:

When journalists delete, dismiss, or disengage from Twitter or Facebook or YouTube or Instagram or Reddit or blogs, they turn their backs on the people who finally-like the journalists - have a printing press to call their own. For too long - since Habermas' alleged birth of the public sphere in the coffee houses and salons of London and Paris - that sphere has excluded too many people, whom social media finally can include. Listen to them. (...) "Twitter is ruining American journalism,” says Manjoo. No, journalists are responsible for the state of American journalism. They have no one to blame but themselves when they jump on a story too soon with unconfirmed information and rash conclusions, when they insist on joining in with their own needless and repetitive hot takes, when they match snark for snark. When I'm a jerk on Twitter it's because I'm being a jerk, not because Twitter made me on.

Como puede verse, Jarvis no admite que la interfaz de las redes sociales determine en alguna medida el flujo de las noticias o la conducta de los usuarios, pero esta postura supone ignorar tanto la existencia de los algoritmos que jerarquizan la visualización de los mensajes como el propio ritmo de lectura/publicación de los mismos.

\section{Nuevos actores: la aparición de las apps de mensajería}

El Informe Reuters sobre periodismo digital para 2018 señala la importancia que han adquirido las aplicaciones de mensajería como WhatsApp en la difusión de las noticias (incluyendo las noticias falsas). Los partidos políticos hacen uso ingente de estas aplicaciones, que se han revelado como decisivas en la propaganda electoral de las últimas contiendas (Caetano et al. 2018; Fares 2018). Klinger-Vilenchik (2016) señalan el valor de los grupos de WhatsApp como espacio que permite la deliberación política y el intercambio cívico de opiniones. La investigación de Karapanos et al (2016) sobre el uso diferenciado de Facebook y de WhatsApp por parte de 464 usuarios, muestra que los usuarios valoran WhatsApp porque ofrece la posibilidad de verdadera interacción, en oposición a las redes sociales, y desbloquea posibles nuevas oportunidades de relación. Por el contrario, Facebook ofrece un uso más satisfactorio en lo referente a estar al día sobre la actualidad y permitir la autoexpresión, pero provoca insatisfacción al exponer a los usuarios a contenidos no deseados. 
Enguix, S. (2019).

Diez años de redes sociales y periodismo: riesgos y retos en el ecosistema informativo del s. XXI pp. 164/183

La integración de este tipo de servicios por parte de la prensa tiene ya algunos años; por ejemplo, Sánchez Gonzales y Sánchez González (2017: 67) señalan que eldiario.es utilizaba un canal de Telegram desde marzo de 2016.

\section{Conclusiones}

En este trabajo hemos ofrecido una panorámica general sobre el impacto tienen desde su aparición las redes sociales en el periodismo político. Las RRSS han modificado de manera radical los procesos de distribución de contenidos informativos de los medios de comunicación tradicionales, y los procesos de producción y elaboración de esos mismos contenidos. Subrayamos que, además, erosionan de manera directa el trabajo de intermediación de los medios; las redes actúan como actores políticos en el ecosistema comunicativo porque, aunque no elaboran directamente contenidos, sí deciden, mediante complejos y opacos algoritmos, qué contenidos puede ver el usuario; y esto incide, directamente, en la percepción que esos mismos usuarios tienen de la realidad política en la que conviven.

El entusiasmo inicial que generaron las redes sociales ha devenido en una sucesión de alertas, tanto para los medios de comunicación como para los usuarios. La extrema dependencia de las RRSS para difundir noticias se convierte en un riesgo no calculado sobre las consecuencias que para un medio pueden tener los citados algoritmos. Y las rutinas productivas están hoy, cada vez más, condicionadas por las propias pautas y márgenes lingüísticos que imponen estas plataformas digitales. La convivencia de las RRSS y los medios de comunicación, y por tanto de los periodistas, ofrece claroscuros que están ofreciendo abundante bibliografía: desde la evidencia de que permiten una mejor segmentación de los públicos objetivos o el refuerzo del perfil del propio periodista, hasta la viralización de noticias falsas, en la que en ocasiones participa el propio informador ante la dificultad de contrastar los hechos dada la velocidad con la que se producen y divulgan los contenidos.

Se trata, en suma, de una profunda transformación de los sistemas tradicionales, con efectos que habrá que valorar regularmente, puesto que estas mismas RRSS, a las que se añade el protagonismo que están adquiriendo los canales de mensajería electrónica (Whatsapp y Telegram, principalmente), inciden directamente en el relato de la información política. Entendemos, que la investigación está acotando cada vez más sus esfuerzos para dirimir hasta qué punto el periodismo político puede ver afectadas sus bases deontológicas por unas dinámicas que ya no dependen del control de los medios de comunicación tradicionales, sino de empresas cuya capacidad tecnológica está muy desarrollada y que, además, disponen de los recursos humanos y tecnológicos para innovar, de manera continua, las aplicaciones que sirven para distribuir y consumir contenidos informativos. No existe, por lo tanto, una conclusión positiva y negativa al uso, pues los efectos de las RRSS en el periodismo político generan multiplicidad de 
Enguix, S. (2019).

Diez años de redes sociales y periodismo: riesgos y retos en el ecosistema informativo del s. XXI pp. 164/183

percepciones, entre profesionales e investigadores. Lo que anima a seguir atendiendo con rigor científico la evolución de una realidad que ha transformado, para siempre, el propio periodismo político.

\section{Referencias}

Ahmad, Ali Nobil (2010): “Is Twitter a useful tool for Journalists?”, Journal of Media Practice 11, pp. 145-155.

Applebaum, Anne (2015): "Mark Zuckerberg should spend \$45 billion on undoing Facebook's damage to democracies", The Washington Post, 10/12/2015, https://www.washingtonpost.com/opinions/mark-zuckerberg-could-spend-45-billionon-undoing-facebooks-damage/2015/12/10/4b7d1ba0-9e91-11e5-a3c5c77f2cc5a43c_story.html

Bell, Emily (2014): “Silicon Valley and Journalism: Make up or Break up?”, Reuters Memorial Lecture 2014, Reuters Institute en Oxford, 21 de noviembre de 2014. https://reutersinstitute.politics.ox.ac.uk/calendar/silicon-valley-and-journalism-make-orbreak

Bell, Emily (2016): "Facebook is eating the world”, Columbia Journalism Review, 07/03/2016, https://www.cjr.org/analysis/facebook and media.php

Bennett, W. Lance \& Iyengar, Shanto (2008): “A new era of minimal effects? The changing foundations of political communication”, Journal of communication 58.4 (2008): 707-731. doi:10.1111/j.1460-2466.2008.00410.x

Bobba, Giuliano (2018): “Social media populism: Features and 'likeability’of Lega Nord communication on Facebook", European Political Science (2018): 1-13. https://doi.org/10.1057/s41304-017-0141-8

Broersma, Marcel \& Graham, Todd (2016): "Tipping the Balance of Power Social Media and the Transformation of Political Journalism”, en Axel Bruns, Gunn Enli, Eli Skogerbo, Anders Olof Larsson, Christian Christensen (Eds), The Routledge Companion to Social Media and Politics. New York and Milton Park: Routledge, 2016, pp. 89-103.

Caetano, Josemar A.; Magno, Gabriel; Cunha, Evandro; Meira Jr, Wagner; MarquesNeto, Humberto T. \& Almeida, Virgilio (2018). "Characterizing the public perception of WhatsApp through the lens of media”, arXiv preprint arXiv:1808.05927.

Carrera Álvarez, Pilar, Sáinz de Baranda Andújar, Clara; Herrero Curiel, Eva \& Limón Serrano, Nieves (2012): "Journalism and Social Media: How Spanish Journalists are Using Twitter", Estudios sobre el mensaje periodístico, 18(1), 31-53. http://revistas.ucm.es/index.php/ESMP/article/view/39353

Casero-Ripollés, Andreu (2010): "Prensa en internet: nuevos modelos de negocio en el escenario de la convergencia”, El profesional de la información, 19 (6), pp. 595-601, DOI: 10.3145/epi.2010.nov05 
Enguix, S. (2019).

Diez años de redes sociales y periodismo: riesgos y retos en el ecosistema informativo del s. XXI pp. 164/183

Casero-Ripollés, Andreu (2012): “Contenidos periodísticos y nuevos modelos de negocio: evaluación de servicios digitales”, El profesional de la información, v. 21, n. 4, pp. 341-346. https://doi.org/10.3145/epi.2012.jul.02Chadwick, Andrew \& Stanyer, James (2010): "Political Communication in Transition: Mediated Politics in Britain's New Media Environment." Political Studies Association Annual Conference. Edinburgh, $U K$. 2010. http://newpolcom.rhul.ac.uk/storage/Chadwick_Stanyer_Political_Communication_in_ Transition APSA 2010 FINAL.pdf

Clavero, Juan (2018): "Posverdad y exposición selectiva a fake news. Algunos ejemplos concretos de Argentina". Contratexto 029, pp. 167-180.

Del Fresno, Miguel (2015): "Internet como macromedio. La cohabitación entre medios sociales y medios profesionales”, Telos 99, pp. 107-109, https://telos.fundaciontelefonica.com/revista/

Daniels, Jessie (2018): “The Algorithmic Rise of the 'Alt-Right””, Contexts 17.1, pp. 6065. https://journals.sagepub.com/doi/full/10.1177/1536504218766547

Fares, Fatima (2018): "WhatsApp y Periodismo. Análisis del uso de WhatsApp en los medios de información españoles”. Hipertext.net: Revista Académica sobre Documentación Digital y Comunicación Interactiva, 16, pp. 7892. http://doi.org/10.31009/hipertext.net.2018.i16.12

Fernández Lombao, Tania y Vázquez Sande, Pablo (2016): “Twitter: ¿Cómo actúan los directores de los principales diarios de España en esta red social?”, Mediatika 15, pp. 101123.

Gallardo Paúls, Beatriz (2017): «Pseudopolítica en la red: indicadores discursivos de desideologización en Twitter», Pragmalingüística 25, pp. 189-210. https://doi.org/10.25267/Pragmalinguistica.2017.i25

Gallardo Paúls, Beatriz (2018): Tiempos de hipérbole. Inestabilidad e interferencias en el discurso político, Valencia: Tirant Lo Blanch.

Gallardo Paúls, Beatriz y Enguix Oliver, Salvador (2016): Pseudopolítica: el discurso político en las redes sociales, Valencia: Dept. Teoria de los Lenguajes y Ciencias de la Comunicación de la Universitat de València.

Greenslade, R. (2016). "Publishers call on government to help over Google and Facebook", The Guardian, 22/09/2016, https://www.theguardian.com/media/greenslade/2016/sep/22/publishers-governmentgoogle-facebook-newspaper

Greensland, Roy (2016): "Why Facebook is public enemy number one for newspapers, and journalism”. The Guardian, 20/09/2016, https://www.theguardian.com/media/greenslade/2016/sep/20/why-facebook-is-publicenemy-number-one-for-newspapers-and-journalism 
Enguix, S. (2019).

Diez años de redes sociales y periodismo: riesgos y retos en el ecosistema informativo del s. XXI pp. 164/183

Guess, A., Nyhan, B., y Reifler, J. (2018): Selective Exposure to Misinformation: Evidence from the Consumption of Fake News during the 2016 US Presidential Campaign. Exeter: Department of Politics.

Hedman, Ulrika \& Monika Djerf-Pierre (2013): “The social journalist: Embracing the social media life or creating a new digital divide?”, Digital Journalism 1.3, pp. 368-385.

Hermida, Alfred (2010). “Twittering the news: The emergence of ambient journalism”. Journalism practice, 4 (3), 297-308. http://dx.doi.org/10.1080/17512781003640703

Jarvis, Jeff (2019): "Journalism is the conversation. The conversation is journalism”, Medium,27/02/2019, https://medium.com/whither-news/journalism-is-the-conversationthe-conversation-is-journalism-22a8c631e952

Karapanos, Evangelos; Teixeira, Pedro \& Gouveia, Ruben (2016): "Nedd fulfillment and experiences on social media: A case on Facebook and WhatsApp", Computers in Human Behavior 55, pp. 888-897, http://dx.doi.org/10.1016/j.chb.2015.10.015

Kwak, H., Lee, C., Park, H. y Moon, S. (2010): "What is Twitter, a social network or a news media?", Proceedings of the 19th international conference on World wide web, ACM, pp. 591-600. https://dl.acm.org/citation.cfm?id=1772751

Liddle, Celeste (2016): "Hate-Speech by another name: Whay the term 'alt-right' should not be legitimised", The Sydney Morning Herald, 25/11/2016, https://www.smh.com.au/lifestyle/altright-is-not-a-thing-and-lets-not-use-...2

López-García, Guillermo (2017a): “Comunicación política y discursos sobre el poder”. El profesional de la información, v.26, n. 4, pp. 573578.https://doi.org/10.3145/epi.2017.jul.01

López García, Guillermo (2017b): “Rápida consolidación de las redes sociales”, en DíazNosti, Bernardo (Coord.): Diez años que cambiaron el mundo, 2007-2017, Barcelona/Madrid: Ariel/Fundación Telefónica, pp. 233-

Majoo, Farhad (2019): "Periodista: es el momento de dejar las redes sociales”, The New York Times, 26/01/2019, https://www.nytimes.com/es/2019/01/26/twitter-dejar-redessociales/?smid=wa-share-es

Maréchal, Nathalie (2016): “Automation, algorithms, and politics| when bots tweet: Toward a normative framework for bots on social networking sites (feature)", International Journal of Communication, 10, pp. 5022-5031.

Nafría, Ismael (2015): “El impacto de internet en la campaña electoral”, La Vanguardia, 11/12/2015,

https://www.lavanguardia.com/politica/elecciones/20151211/30724829901/impactointernet-campana-electoral.html

Newman, Nic; Fletcher, Richard; Kalogeropoulos; Levy, David \& Nielsen, Rasmus K. (2018): Reuters Institute Digital News Report 2018, https://reutersinstitute.politics.ox.ac.uk/sites/default/files/digital-news-report-2018.pdf 
Enguix, S. (2019).

Diez años de redes sociales y periodismo: riesgos y retos en el ecosistema informativo del s. XXI pp. 164/183

Palacio, Luis (2018): "Modelos de negocio de los medios digitales: diversificar ingresos para financiar el periodismo”, Cuadernos de periodistas 36, pp. 9-22. http://www.cuadernosdeperiodistas.com/media/2018/07/9-22-Luis-Palacio.pdf

Peiser, Jaclyn (2019): “The New York Times Co. Reports \$709 Million in Digital Revenue for 2018”, The New York Times, 06/02/2019, https://www.nytimes.com/2019/02/06/business/media/new-york-times-earnings-digitalsubscriptions.html

Pérez, Víctor (2018): “Cuánto dinero ganan Facebook, Instagram, Twitter y Google con tus datos personales”, Business Insider 22/07/2018 https://www.businessinsider.es/estoes-dinero-que-ganan-facebook-instagram-twitter-google-tus-datos-personales-198114

Reguera, Marcos (2017): “Alt Right: radiografía de la extrema derecha del futuro”, CTXT, Contexto y Acción, 22 de febrero de 2017. http://www.pensamientocritico.org/marreg0917.pdf

Revers, Matthias (2014): "The Twitterization of News Making. Transparency and Journalistic Professionalism”, Journal of Communication 64.5: 806-826. http://onlinelibrary.wiley.com/doi/10.1111/jcom.12111/abstract

Salmon, Felix (2016): "Mark Zuckerberg just made his boldest power grabe ever", Splinter, 29/04/2016, https://splinternews.com/mark-zuckerberg-just-made-his-boldestpower-grab-ever-1793856510

Sánchez-Gonzales, Hada; Sánchez-González, María (2017): “Los bots como servicio de noticias y de conectividad emocional con las audiencias. El caso de Politibot”. Doxa Comunicación, $\quad$ n. $25, \quad$ pp. 63-84. http://dspace.ceu.es/bitstream/10637/8765/1/Bots HadaSanchez MariaSanchez Doxa 2017.pdf

Theocharis, Yannis; Barberá, Pablo; Fazekas, Zoltán \& Popa, Sebastian (2016): “Twitter trolls are actually hurting democracy”, The Washington Post, 4/11/2016.

Tumasjan, Andranik, et al. (2010): "Predicting elections with twitter: What 140 characters reveal about political sentiment", Proceedings of the Fourth International AAAI Conference on Weblogs and Social Media, Icwsm 10.1, pp. 178-185. https://www.aaai.org/ocs/index.php/ICWSM/ICWSM10/paper/viewFile/1441/1852 